\title{
The Design and Implementation of Laboratory Management System based on Internet of Things
}

\author{
Zhengbo $\mathrm{Li}^{1 \text {, a }}$ \\ ${ }^{1}$ School of Economic Management, Beihua University, Jilin 132013, China. \\ a zhengbo_li@126.com
}

Keywords: laboratory management, Internet of Things, intelligent monitoring.

\begin{abstract}
According to current situation of university laboratory management and development of the Internet of Things, the system based on the Internet of Things for laboratory management is designed for intelligent management. The system combines the technology of Internet of Things and Mobile Internet. Tests show that the system meets the needs of university laboratories and reaches the commercial level and achieves automatically management of information as well as intelligent monitoring.
\end{abstract}

\section{Introduction}

In recent years, with people's emphasis on education, higher education flourish, enrollment in colleges and universities are also increasing. With the global development of information technology, new subjects, new specialties are emerging. In information technology, "Twelfth Five-Year Plan", the state put forward the slogan of building "smart campus", hoping to twenty-first century university building into information, intelligence university. In this campus information, intelligence wave, university laboratory information, intelligence has become a key university construction.

University Laboratory management mostly through traditional artificial way management method not only backward, cumbersome and laborious, inefficient and costly. Traditional management methods already unable to meet current development laboratory, the current status of the management of specific performance is as follows:

The traditional way using manual registration apparatus, generally require regular equipment procurement and inventory updating inventory every year, not only the heavy workload, prone to error, and not a good real-time query the device information, supervision and maintenance. So often missing equipment, laboratory administrator to great trouble, from the point of view of the school, causing unnecessary losses to the school property.

The traditional method of artificial statistics student information when the student's phone, mail and other personal information changes, you can not immediately update to the students' personal information. In addition, each semester laboratory student mobility than larger workload, cumbersome. To laboratory management personnel caused great trouble. On the experimental class, the experimental class student assistants need to count attendance, the traditional manual way, cumbersome and inefficient. Since there is no common shared platform, sharing the same nature between laboratory resources is low, especially in some high-end sophisticated equipment, laboratory equipment across complex procedures. Traditional laboratory door, curtains and lighting are key mechanical and manual mode switch, not enough intelligence and information can not be remote controlled. Not a complete record of laboratory usage. No traditional laboratory environment monitoring system, can not be found that the disaster in case of fire and other disasters occur, it can not be found, giving the laboratory a devastating loss.

With the rapid development of microelectronics, micro systems technology, communications technology and network technology, the late 1990s put forward the concept of things. Things is based on an existing computer on the Internet, the use of micro-electronics technology, RFID radio frequency technology, self-organizing network technology structure contains a lot of kind of communication network. In the middle of things, things and things, between objects and people can communicate with each other. The 21st century is the era of communications networking, the 
ultimate goal is to achieve things smarter planet, which is the development trend of things, but also the development trend of human society. The idea has been in the past people exchanges and communication between people, things realized things and things, exchange and communication between people and objects. On the basis of things that people can be more comfortable and conveniently manage their production and life. In order to solve the current university laboratory management issues that arise, given the years of development of networking technology and smart home applications mature, the Internet of Things technology to laboratory management has matured.

With the rapid development of information technology, especially the development of powerful networking technology and mobile Internet technology for the intelligent development laboratory provides a golden opportunity for the realization of university laboratory automation, information technology and intelligent management to provide strong technical support. For the above, based on the development of the Internet of Things laboratory management system, enabling the laboratory environment, weather monitoring and scientific management of information flow.

\section{Internet Technology}

Things That network is connected to all things, including things and things connected with the people was connected. Things mainly has the following characteristics: First, the ability to have things information collection and identification, that things get information through a variety of sensors or RFID radio frequency identification technology; again, things is also a network the ability to have a network to transmit data, things must have the ability to deliver real-time information on the Internet; and finally, the Internet of things through massive data analysis and processing, the ability to intelligent control.

Perception layer is mainly composed of some perception module (such as card readers, sensors, RFID radio frequency identification and cameras), the network module (such as ZigBee networks, etc.) and a controller. Perception layer node is responsible for collecting sensory information (such as temperature and humidity, PM2.5, light, video, card information, etc.), and finally by the gateway to upload information self-organizing wireless sensor networks to collect the information sent to the gateway, to the host computer. Such as the current popular smart home, industrial monitoring applications are using this technology.

Things network layer is different from the network layer of the OSI model of the Internet, the network layer things include not only Internet communications networks, also includes a wireless communication network. Any access to the Internet operations are part of a network of things. Mainly through the application layer analysis and processing of information collected sensing layer to provide convenient services to users based on the analysis results such as smart home is through a variety of sensing devices collect information inside the home to provide convenient services to people's home life. Things applications in the topmost layer, application layer services directly to customers. Developers can be customized to provide customers with a wide range of specific services. Application layer is the core networking technology, application-layer technology directly affects Internet of Things technology development and diffusion.

\section{System Requirements Analysis}

Based on business objectives IOT lab management system to replace the current manual management mode of university laboratory, a laboratory environment to add real-time monitoring and intelligent control and other functions, so that the laboratory data management information, simplifying laboratory workflow management, improve the technological level of laboratory management, providing strong support for the laboratory information and intelligence. End-use of the system to achieve intelligent management of the laboratory, to implement the "smart campus" concept of modern university. Specific objectives are as follows:

For personnel and equipment, the laboratory personnel replace the old manual registration and information management device information, laboratory information management resources to 
facilitate the sharing of laboratory resources, equipment and personnel information to add, delete and modify graphical query, analysis and statistics of the interface.

For environmental monitoring laboratory, from scratch, using networking technology, laboratory temperature, humidity, PM2.5 and illumination parameters for real-time monitoring, to provide a friendly interactive interface, to achieve the laboratory temperature, humidity, Discover PM2.5 and light graphical interface, analysis and statistics.

\section{Establishment structure}

void set_small_edges_faces_tol(double \&face_tol,double \&edge_tol);

void user_function_for_healing_workflow(ENTITY_LIST\& bodies_imported_into_ACIS,ENTITY_LIST\& output_bodies,double heal_tol);

double user_function_to_determine_max_stitch_tol(double heal_tol);

void user_function_to_stitch_entities(ENTITY_LIST\& to_be_stitched, ENTITY_LIST\& output_bodies,tolerant_stitch_options\& sopts);

double user_function_to_determine_desired_gap_tightness(double heal_tol);

int adjust_ff_sliver(FACE *ff,ENTITY_LIST \&sliver_faces);

void detect_small_faces(ENTITY_LIST \&list,ENTITY_LIST \&sliver_faces,double face_tol);

void detect_and_delete_small_edges(ENTITY_LIST \&savelist,ENTITY_LIST \&short_edges,double edge_tol);

int RAO_HealingEntity( ENTITY_LIST \& oldlist, ENTITY_LIST \& newlist ) ;

inline int adjust_ff_sliver(FACE *ff,ENTITY_LIST \&sliver_faces)

\{

int sign $=0$;

for(int $\mathrm{i}=0 ; \mathrm{i}<$ sliver_faces.count();i++)

\{

FACE $*$ s $=($ FACE $*)$ sliver_faces[i];

if $(\mathrm{ff}==\mathrm{s})$

\{

sign $=1$;

\}

break;

\}

return sign;

\}

inline void set_small_edges_faces_tol(double \&face_tol,double \&edge_tol)

\{

face_tol=0.05;

edge_tol=0.05;

\}

inline void detect_small_faces(ENTITY_LIST \&list,ENTITY_LIST \&sliver_faces,double face_tol)

\{

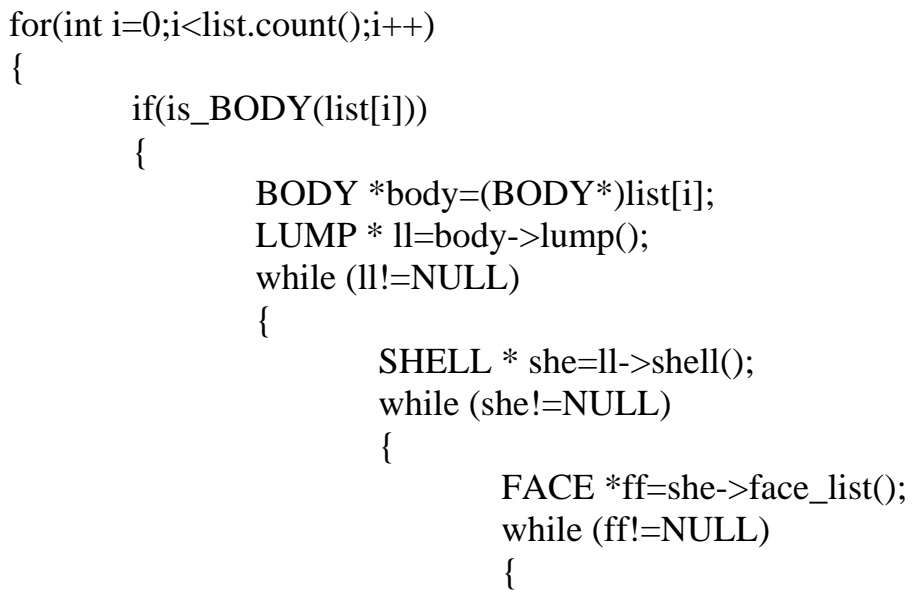




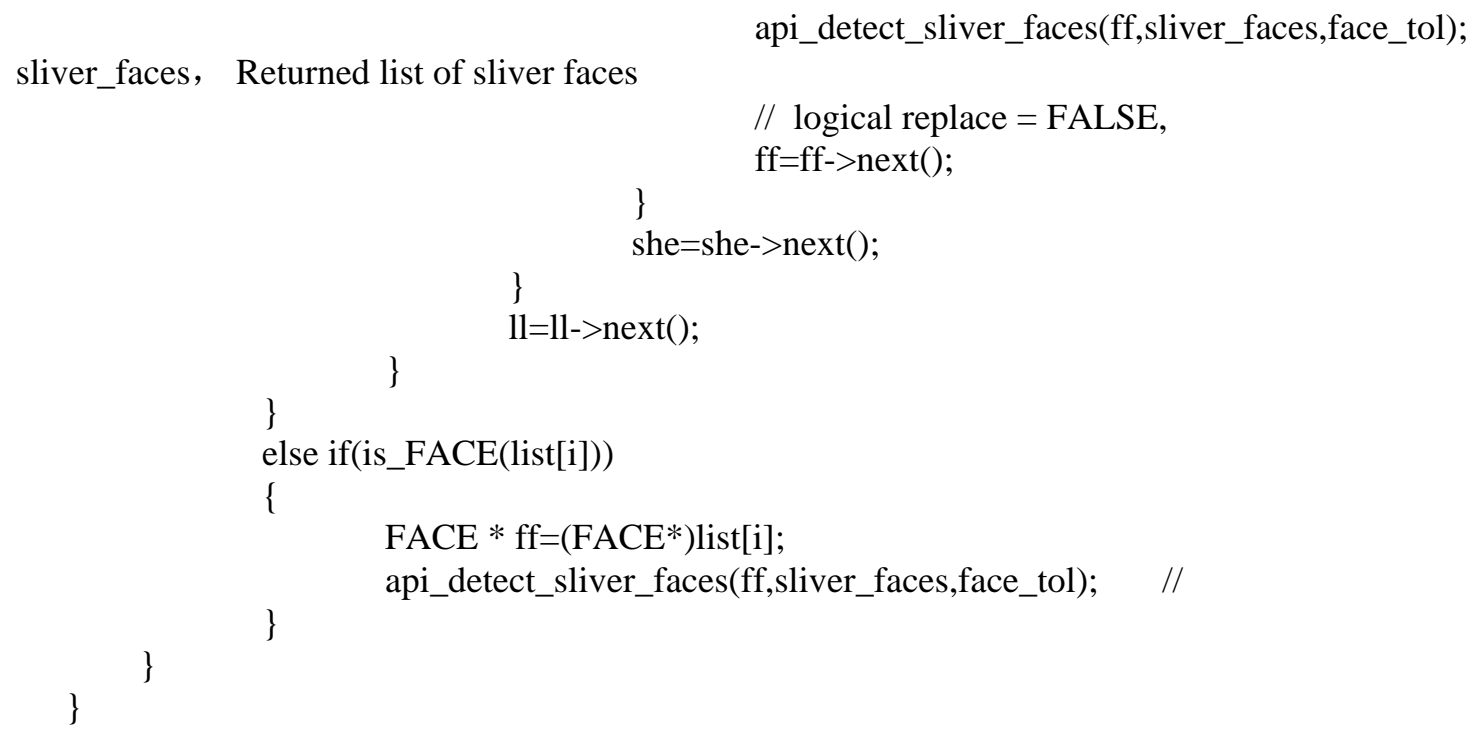

\section{Conclusion}

According to the existing success stories, through access to a lot of things like mobile Internet and related technology information, combined with the actual needs of the university laboratory, and put forward the overall design scheme of Things and mobile Internet technology combined Laboratory Management System .

Designed and implemented throughout the laboratory information management server and the monitoring system, the server uses the popular Struts 2 architecture, data using open-source MySQL database.

Designed and implemented based on Struts 2 technology, MySQL database and Bootstrap Technology Laboratory Management through Internet sites. The site includes user management, device management, node management, environmental management, intelligent control and management and attendance management module. Designed and implemented based on the Android platform mobile client, mobile client to achieve remote door, switch light curtains and switching functions.

\section{References}

[1] Ansai Things smart appliances Design Trend Zhengzhou: Zhengzhou University of Light Industry, 2010.

[2] Wangle Yan, Yuan Liqing, networking technology and its development prospects Inner Mongolia Science Technology and Economy, 2013 (12): 66-68.

[3] Guo Fang, Zhangdong Xia networking technology status of scientific and technological aspect was China, 2013 (1): 79-80.

[4] Xiong Ying mobile Internet and networking development trend and outlook chapter of 2013 (21): 298-299.

[5] Zhu Hongbo, Yanglong Xiang, Zhu Qi networking technology progress and application of Nanjing University of Posts and Telecommunications, 2011 (31): 1-9.

[6] Liu Sang database technology base and application Beijing: Electronic Industry Press, 2003.

[7] Sa Shi Xuan, Wang Shan Introduction to Database Systems Beijing: Higher Education Press, 2000. 